\title{
Uso de la revista digital como herramienta didáctica en los centros de secundaria
}

Silvia Tejada ${ }^{a, b}$, Samuel Pinya ${ }^{c}$, Xavier Capód ${ }^{d}$ Antoni Sureda ${ }^{b, d}$

${ }^{a}$ Laboratory of Neurophysiology, Department of Biology, University of the Balearic Islands, E-07122 Palma de Mallorca, Spain, silvia.tejada@uib.es. ${ }^{b}$ CIBEROBN (Physiopathology of Obesity and Nutrition), E-07122 Palma de Mallorca, Balearic Islands, Spain, ${ }^{\mathrm{C}}$ Interdisciplinary Ecology Group, Biology Department, University of the Balearic Islands, E-07122 Palma de Mallorca, Spain, s.pinya@uib.es, ${ }^{\mathrm{d}}$ Research Group on Community Nutrition and Oxidative Stress (NUCOX), University of Balearic Islands, E-07122 Palma de Mallorca, Balearic Islands, Spain, xaviercapofiol@hotmail.com, antoni.sureda@uib.es

Abstract

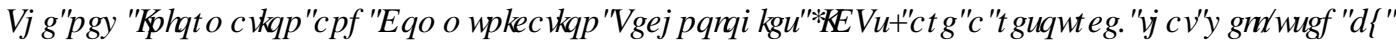

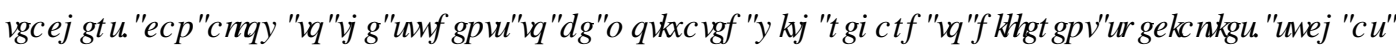

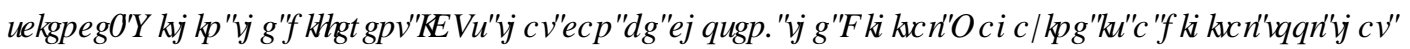

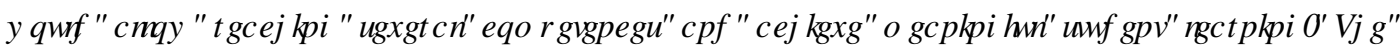

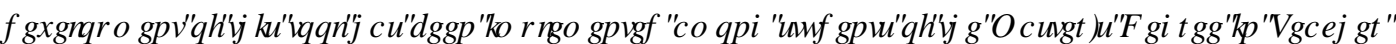

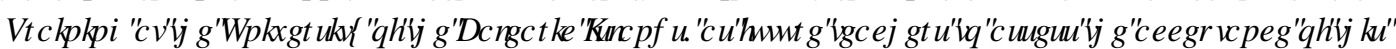

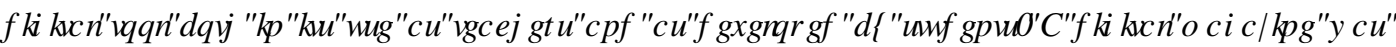

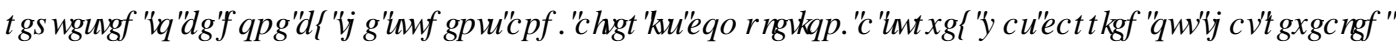

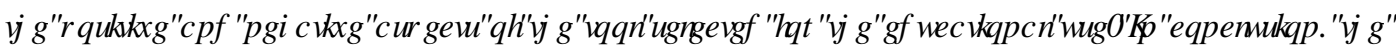

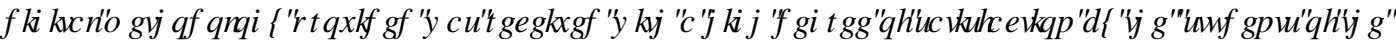

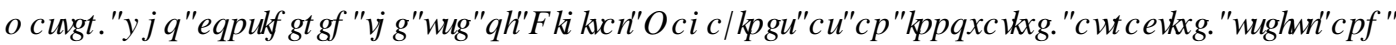
P RUYDUQJLGJLWDOUHRXIFHIRUUYFQQDUIVFRROMXGHQWD

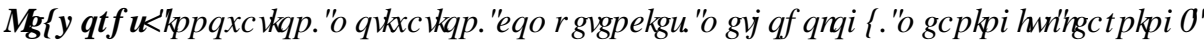

]

\section{HXP HQ}

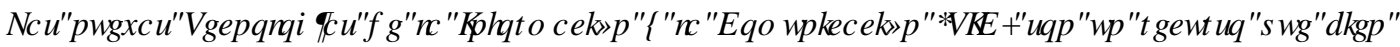

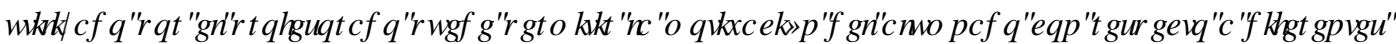

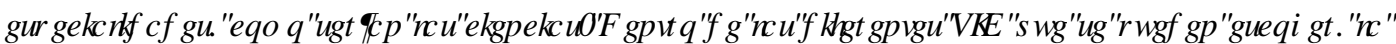

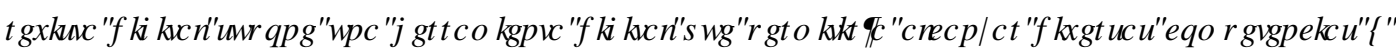



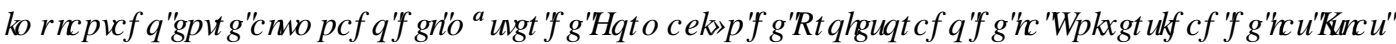

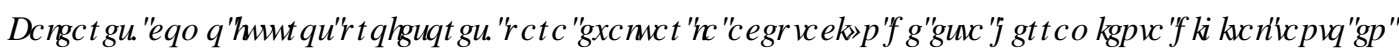

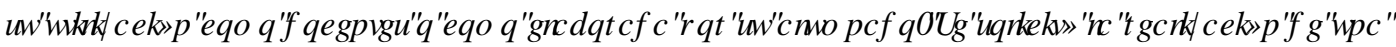

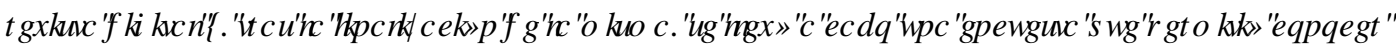

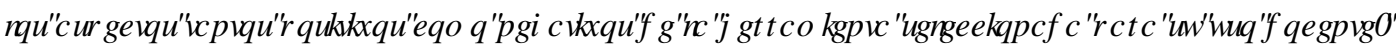

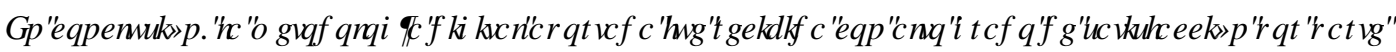

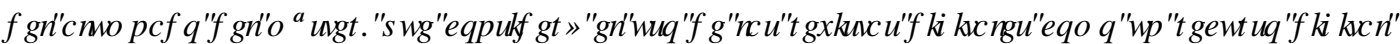

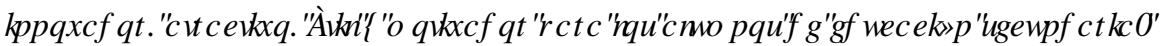






\section{Introducción}

En la actualidad, la educación se basa en muchos casos en un aprendizaje tradicional de los conceptos incluidos en los currículums, de tal manera que el profesor es el eje central que facilita la información que se quiere que los alumnos asuman. Esto hace que el alumno se convierta en un mero espectador y no se consiga un aprendizaje significativo en el que el alumno sea el actor principal y aprenda a resolver las situaciones a las que se enfrenta (Naranjo, 2009). Adicionalmente, desde hace años el aprendizaje de las ciencias es cada vez menos atractivo para los alumnos, los cuales optan por centrarse en otros aspectos dejando relegado el ámbito científico, el cual es básico para formar a futuros ciudadanos que contribuyan al desarrollo de un país (Solbes et al., 2007). Las nuevas Tecnologías de la Información y la Comunicación (TIC) suponen hoy en día un factor de motivación a nivel docente (Hernández, 2017) no solo para el profesorado que trabaja con nuevas herramientas impensables hace unos años, sino también para el alumnado a todos los niveles educativos. Este aspecto es importante dada la falta de motivación que se puede encontrar dentro de las aulas para el aprendizaje de conceptos y habilidades y convierte al profesor en un acompañante del alumno que lo guíe en su proceso de aprendizaje. Hay que añadir que las tecnologías digitales se han desarrollado a un ritmo vertiginoso provocando grandes cambios en el proceso educativo (Busquet-Duran et al., 2013). Además, las TIC suponen una gran influencia dentro de nuestra sociedad actual, por lo que conocerlas y saber aplicarlas dentro del ámbito científico supone una garantía de aceptación por parte de la sociedad en general, y más específicamente, por parte del alumnado, que deberá adaptarse a su uso para su futura inserción en la vida laboral y social en su vida adulta (Amores, 2019). Las TIC en educación permiten fomentar diversas competencias establecidas en el currículum, claramente la competencia digital es la primera de ellas, y no solo en el alumnado sino también en el propio profesorado permitiendo una innovación y mejora en la calidad educativa (Aguilar, 2012); otra competencia importante que se consigue con el fomento de estas nuevas herramientas sería la lingüística (donde se podrían trabajar con un idioma concreto y/o integrar diferentes idiomas).

Dentro de las diversas opciones tecnológicas al alcance de la docencia, las revistas digitales son una de las herramientas que permiten conseguir la motivación y el interés por ambos, profesorado y alumnado. Se trata de una herramienta atractiva que puede desarrollarse según la propia iniciativa y creatividad de los alumnos, permitiendo un desarrollo de su imaginación y que permite el debate y la participación entre compañeros, estableciendo un proceso de retroalimentación para incrementar el conocimiento. Además, es una herramienta que permite que el alumno sea autónomo desarrollando la actividad en cualquier escenario y en diversos soportes de su elección. De esta manera se consigue una mayor implicación que permite profundizar en los conceptos y temas que se quieren trabajar en clase, fomentando la utilización de fuentes bibliográficas diversas. En este enclave, el profesor pasa de ser el actor principal a ser un facilitador del aprendizaje del alumnado, ya que será el mediador para la organización que se debe establecer para el buen funcionamiento de la herramienta digital, así como del material didáctico que el alumnado o el profesorado mismo pueda incorporar en la revista y en el desarrollo de las capacidades de alumnado (Tello y Aguaded, 2009). Por lo tanto, se trata de conseguir un aprendizaje significativo basado en la tecnología en el que el alumnado desarrolle diversas competencias, adquiriendo al mismo tiempo los conceptos necesarios según el currículum de una manera más atractiva, motivadora y facilitadora de adquisición de esos conocimientos.

\section{Objetivos}

El objetivo principal del presente proyecto es introducir y evaluar una herramienta digital, concretamente la revista digital, para el desarrollo del aprendizaje significativo, permitiendo la autonomía, creación y discusión grupal que permita fomentar la discusión y el trabajo en grupo y colaborativo, así como el enriquecimiento de ideas y el pensamiento crítico entre el alumnado. Dentro de este objetivo genérico, se evaluará principalmente cómo se valora el uso de esta herramienta por parte de alumnos del máster de 
profesorado de la especialidad de la Biología y Geología, los cuales podrán aplicar dicho conocimiento en su futura vida laboral. Adicionalmente, de manera secundaria se conseguirá mejorar la capacidad de expresión escrita y la motivación por parte del alumnado para alcanzar diferentes aspectos del currículum y diversas competencias.

\section{Desarrollo de la inovación}

La primera parte del proyecto se basó en la preparación de una revista digital a modo ejemplo para que el alumnado del Máster de Formación al Profesorado en la especialidad de Biología y Geología (Universidad de las Islas Baleares, UIB) pudiese conocer las herramientas disponibles (vídeos, gifs animados, links a webs de importancia, etc) y el formato digital para la creación de una revista digital. Se utilizó la plataforma

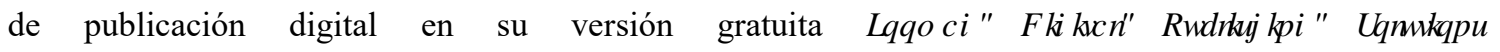
(https://www.joomag.com/es), que permite la creación de contenido interactivo.

Se creó un link dentro de la plataforma Moodle de la UIB para que los alumnos tuvieran acceso a la plataforma de la revista y se les entregó las pautas necesarias para iniciar la creación digital de la revista, para cuyo desarrollo tuvieron un período de 3 meses. Los alumnos se organizaron de dos en dos para la elaboración de este material docente digital indicándose que, en el caso que ocupaba, la revista la realizaba el profesor hacia el alumnado, pero que la mayor implicación de conocer la herramienta era su aplicación en las aulas y que los propios alumnos de secundaria la realizaran y diesen formato. Se hizo hincapié en profundizar en algún tema concreto del currículum de Biología y Geología para conseguir profundizar en temas que podrían ser limitantes dentro de las aulas en su modo clásico de enseñanza. También se elaboró una rúbrica para la evaluación del trabajo realizado por los alumnos, en la cual se debía incluir una portada, un índice, y al menos 6 páginas dedicadas a información relevante sobre el tema libre seleccionado. Con la rúbrica se valoraba punto a punto los aspectos más relevantes que se querían alcanzar con la utilización de esta herramienta digital, como la creación de una portada atractiva y con información relevante del contenido para captar a un mayor número de lectores, unos contenidos adecuados al nivel al que iba destinada la revista así como los recursos que se utilizaban que permitieran un entendimiento de la materia seleccionada y/o su relación con el entorno real del alumnado, o la utilización de otras TICs gracias a que el formato de esta revista permite la edición de contenido interactivo.

Finalmente, se diseñó una encuesta evaluadora (con un total de 20 ítems) para que los alumnos, futuros profesores de secundaria, la pudieran cumplimentar con el objetivo de conocer la aceptación de esta metodología digital, la cual permitiría una mayor comprensión de los conceptos teóricos y actitudinales por parte de sus posibles futuros alumnos en centros de educación secundaria; otro de los objetivos, además, fue poder detectar los aspectos más débiles con la finalidad de poder mejorar esos aspectos en un futuro. Esta encuesta se realizó una vez los alumnos habían entregado la revista realizada por cada pareja para poder evaluar tanto los aspectos positivos como los negativos con los que se habían encontrado a la hora de la elaboración de la misma. 


\section{Resultados}

La propuesta de elaboración de revista digital fue muy buen recibida por el alumno del máster de Formación al Profesorado, el cual presentó propuestas de revistas digitales muy atractivas y con material educativo adecuado a los niveles educativos seleccionados (Figura 1).
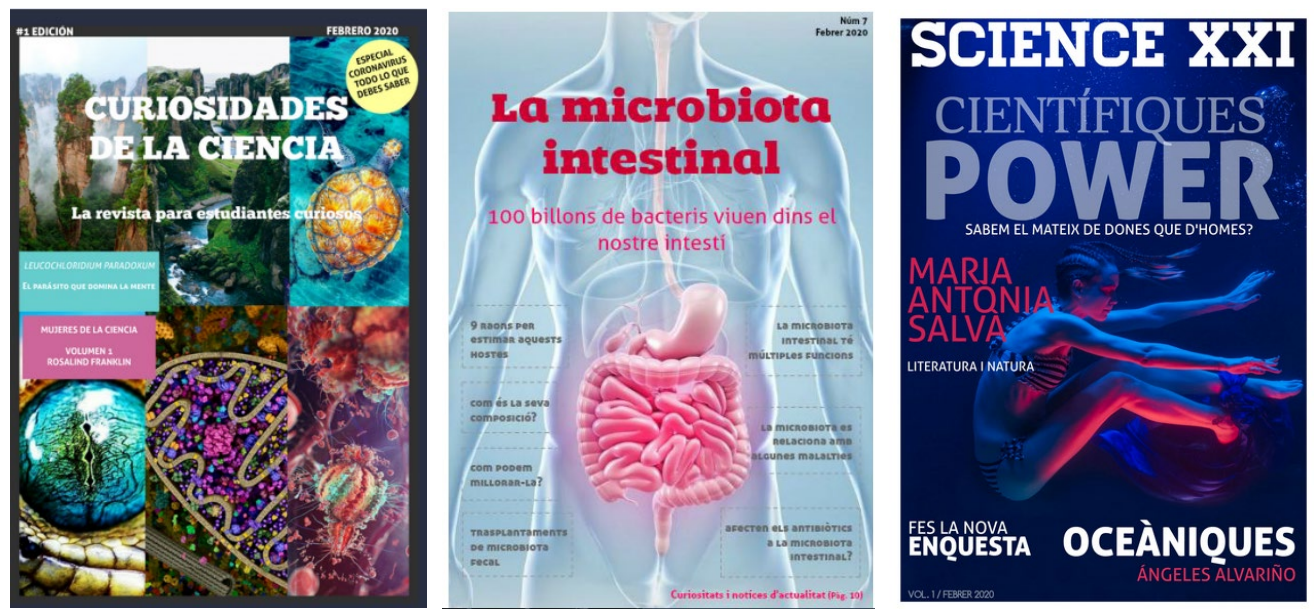

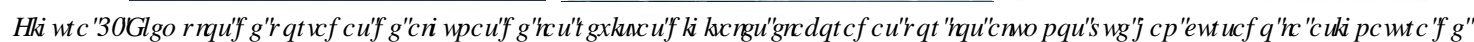

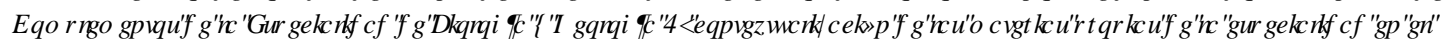


3 XEQMKQJ[6ROWRQV]

La encuesta que se realizó entre el alumnado con la finalidad de conocer los aspectos más fuertes y débiles de la revista digital como herramienta de innovación docente permitió conocer el grado de satisfacción en relación a esta metodología. La misma incluía 20 cuestiones, entre las cuales las respuestas eran categóricas o bien de respuesta libre, mientras que otras preguntas se debía valorar del 0 al 10 siendo el 0 estar en total desacuerdo y el 10 estar totalmente a favor. Las cuestiones de la encuesta son las siguientes:

1.- ¿Cómo evaluarías s el uso de la revista digital como herramienta didáctica preparada por el profesor?

2.- ¿Cómo evaluarías s el uso de la revista digital como herramienta didáctica preparada por el alumno?

3.- ¿La creación de la revista digital te ha permitido incluir diferentes aspectos didácticos y curriculares?

4.- ¿Crees que se pueden conseguir alcanzar diversas competencias gracias a la creación de la revista digital?

5.- ¿Qué competencias intentarías alcanzar con la creación de la revista digital creada por ti como profesor?

6.- ¿Qué competencias intentarías alcanzar con la creación de la revista digital creada por el alumnado?

7.- ¿Cómo valorarías la participación del alumnado en la creación de la revista digital?

8.- ¿Te parece adecuado el uso de una revista digital para reforzar y/o profundizar algunos conceptos de las clases magistrales?

9.- ¿Preferirías la realización de una revista digital monográfica centrándose en la explicación de un tema concreto o una revista digital multidisciplinar/multitemática? ¿Por qué?

10.- ¿Crees que la revista digital podría captar la atención del alumnado en el tema propuesto?

11.- ¿Crees que la revista digital permitiría una mayor búsqueda de respuestas por parte del alumnado en un tema propuesto? (indagación y aprendizaje) 
12.- ¿Realizarías la primera edición de una revista digital como herramienta didáctica para el área de Biología y Geología exclusivamente?

13.- ¿Realizarías la segunda y siguientes ediciones de una revista digital como herramienta didáctica para el área de Biología y Geología en conjunto con otras áreas?

14.- ¿Te parece una buena herramienta para introducir diversos recursos web y docentes?

15.- ¿Qué tipo de recursos principalmente incluirías en una revista digital?

16.- ¿Crees que la creación de una revista digital por parte de tus alumnos permitiría una mayor cohesión de grupo?

17.- ¿Crees que la creación de una revista digital por parte de tus alumnos permitiría una mejora a la atención a la diversidad?

18.- ¿Crees que la creación de una revista digital por parte de tus alumnos ayudaría a conseguir trabajar y alcanzar los objetivos del currículum?

19.- Enumera los aspectos positivos del uso de una revista digital como herramienta didáctica.

20.- Enumera los aspectos negativos del uso de una revista digital como herramienta didáctica.

Los resultados obtenidos en relación a las cuestiones cuantitativas se representan en la figura 2. Los alumnos que participaron en la experiencia fueron la totalidad del grupo, ya que la asistencia era obligatoria, ascendiendo a un total de 24 alumnos. Se puede observar que los promedios en la valoración de las preguntas con respuestas nominales, el valor está por encima de 7, con la excepción de las preguntas 1, 12 y 17. Estas preguntas están relacionadas con la creación de la revista digital por parte del propio profesor, obsevándose un valor promedio de 6,8 $\pm 0,4$ en contra de la elaboración de la misma por parte de los futuros alumnos, con un valor de 7,5 $\pm 0,3$; o con la puesta en marcha de esta herramienta digital exclusivamente como parte de la asignatura de Biología y Geología $(5,6 \pm 0,6)$; así como la utilización de la revista digital en relación a una mejora de atención a la diversidad $(6,8 \pm 0,4)$. Destacar que la mayor puntuación obtenida ha sido para la pregunta que relaciona la consecución de diversas competencias a través del uso de esta herramienta digital por parte del alumnado (pregunta 4; 8,2 $\pm 0,3$ ). 


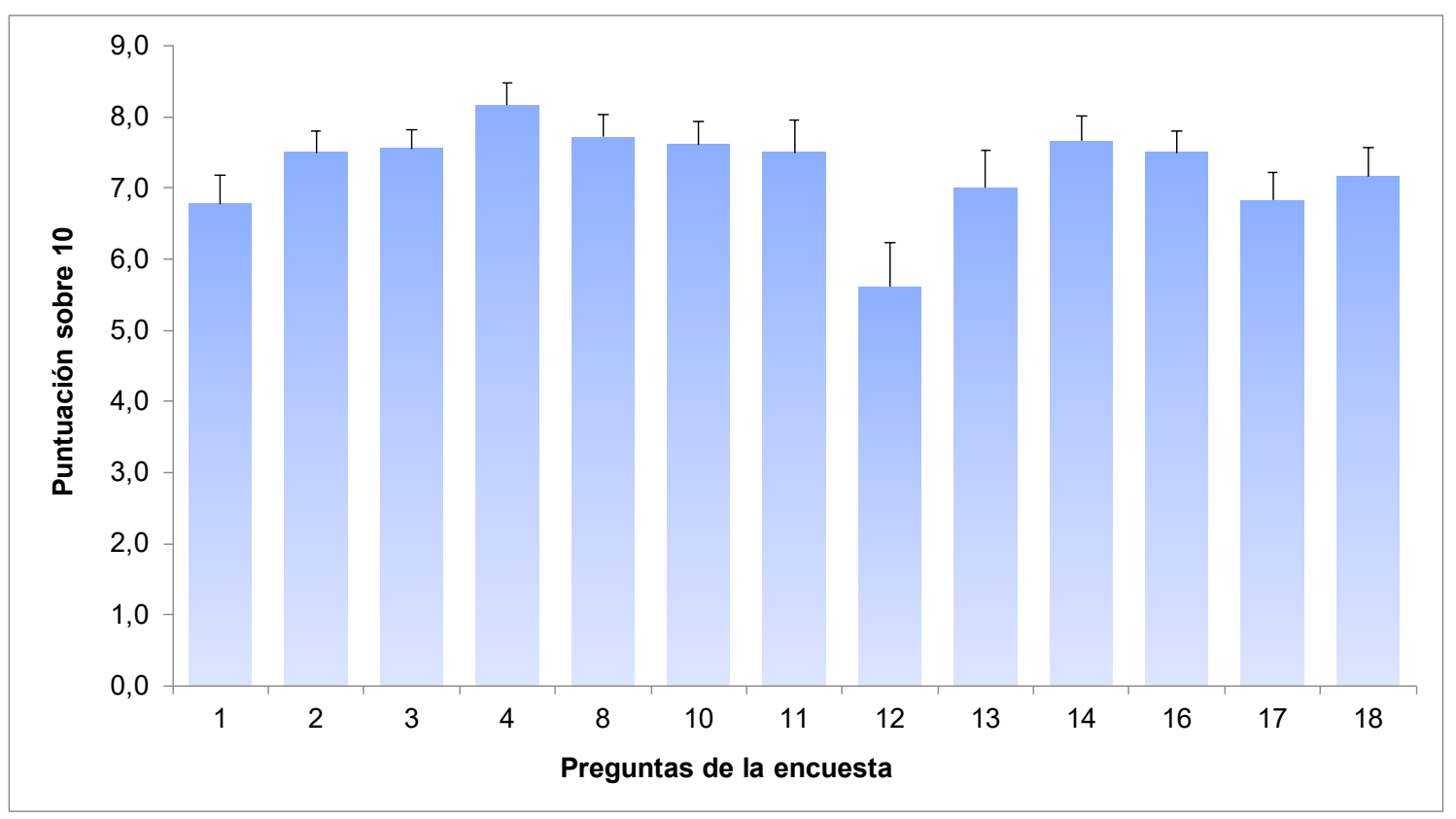

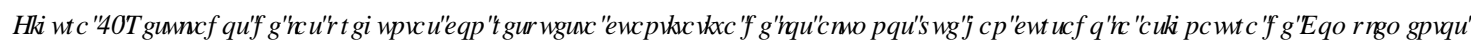

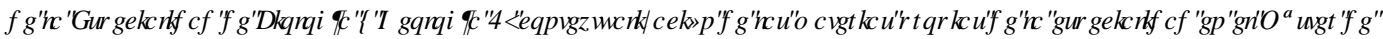

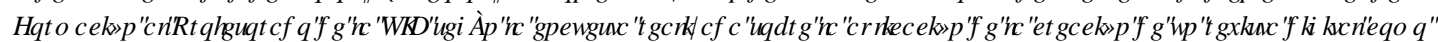

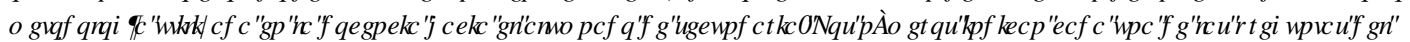 FXHMRQDURIFRQMMDORISRUTRVDOP QRW}

Entre los comentarios de respuesta libre en el cuestionario, se indica cuáles son las competencias que los alumnos del máster creen que podrían conseguir alcanzar con el uso de la revista digital como herramienta didáctica (Figura 3). Se puede observar como tanto si la revista es elaborada por el profesor (pregunta 5 del cuestionario, Figura 3A) como por el alumno (pregunta 6 del cuestionario, Figura 3B), la principal competencia es para la digital seguida por la competencia de aprender a aprender. La competencia científica queda en tercer lugar en el caso de que sea el profesor el que cree la revista digital, mientras que si es el alumno, la competencia lingüística quedaría por delante. Destacar que aunque en unos porcentajes más pequeños, también se han incluido las competencias emprendedora y creativa en ambos casos. Finalmente indicar que en un 5,6\% se ha indicado que se podrían alcanzar prácticamente todas la competencias establecidas en el currículum. 




B

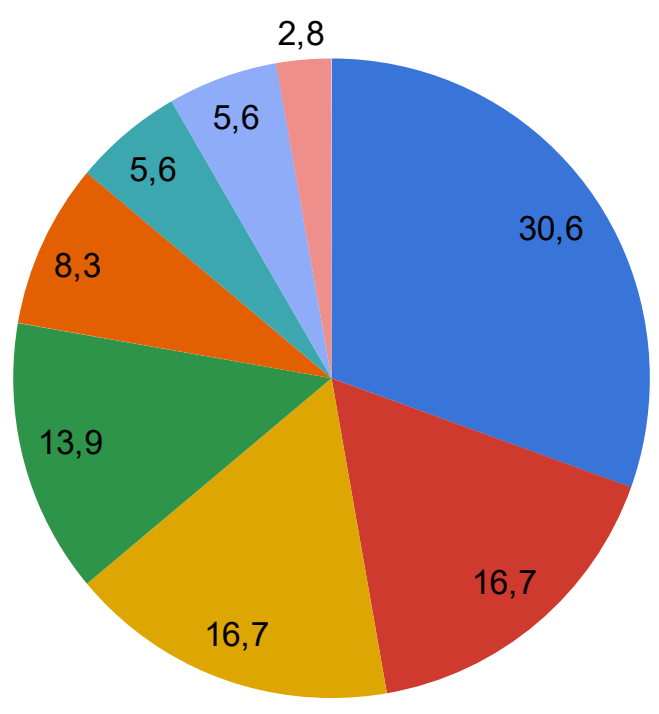

Digital

Aprender a aprender

- Científica

- Comunicación lingüística

- Cívica, social y cultural

Emprendedora

Prácticamente todas

Creativa

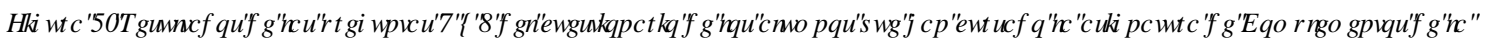

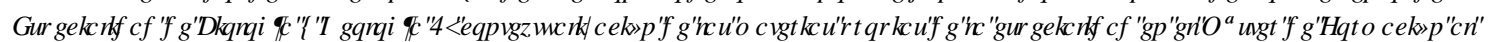

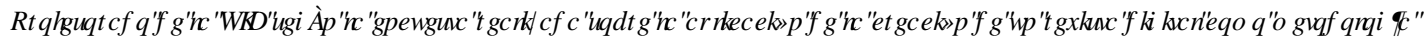

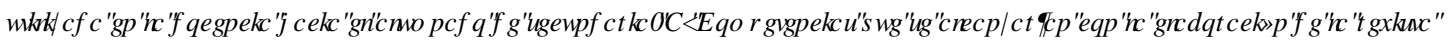


$D O P Q D A R]$

Como se ve la competencia digital tiene un gran peso, tanto si la revista es creada por el profesor como por el alumnado, ya que permite la inclusión del propio temario del currículum con una mejor contextualización así como de diversas TICs adicionales que los mismos alumnos del máster indican (vídeos, imágenes, mapas conceptuales, webs de información de interés para el alumnado, podcasts, artículos científicos y de divulgación, y libros online). Además, los alumnos encuestados indican que valorarían muy positivamente la realización de la misma por parte de sus alumnos y generarían rúbricas de evaluación para evaluarlos durante todo el proceso de creación, además de realizar una coevaluación y autoevaluación. En relación a

(c)) BY-NC-ND 2021, Universitat Politècnica de València 
la creación de una revista monográfica o multidisciplinar, no existe un consenso claro, ya que el $40 \%$ del alumnado indicó que la preferiría monográfica, otro $40 \%$ multidisciplinar y un $20 \%$ valoraría las dos opciones; las razones para una y otra son diversas, como que la realización de una revista digital monográfica permitiría profundizar en un tema concreto haciendo más "expertos" a los alumnos concentrando mejor los esfuerzos y pudiendo seguir una misma línea de trabajo más fácilmente, además de poder completar las unidades didácticas; los alumnos a favor de la multidisciplinariedad avogan por un mayor refuerzo en diversos aspectos del currículum pudiendo ampliar la información en diferentes temas.

Finalmente, en relación a los aspectos positivos en la elaboración de la revista digital se incluyen aspectos como una "metodología innovadora y útil capaz de motivar a los alumnos trabajando el temario de forma más lúdica, entretenida y atractiva" que permite ir estudiando el currículum durante la creación de la misma acercándolo a la realidad y a los familiares y amigos. Si se trabaja de manera conjunta, además, permite una mayor cohesión de grupo y favorecer el trabajo cooperativo involucrando a los diferentes integrantes en la búsqueda de información y en cómo plasmarla y motivándose entre ellos. Indican que "los alumnos son los responsables de su aprendizaje y son activos en este", con lo que se pueden llegar a alcanzar prácticamente todas las competencias según el enfoque que se le de a la herramienta digital. En último lugar, uno de los encuestados resaltó su carácter ecológico, tan importante en la formación actual de los futuros ciudadanos. Por otro lado, también se encontraron diversos aspectos negativos; sobretodo destacar el tiempo que se requiere para la elaboración de este material digital y disponer de los recursos necesarios; además, se indicó que la aplicación utilizada era algo compleja en el momento de su puesta en marcha, aunque también se apuntó a que existe una buena ayuda en línea para resolver los problemas.

\section{Conclusiones}

La introducción de la revista digital como herramienta didáctica y nueva metodología activa de aprendizaje fue valorada de manera general positivamente. La elaboración de dichas revistas permite un trabajo colaborativo en el que sus integrantes pueden exponer sus ideas después de realizar un trabajo de indagación sobre las posibles "noticias" a incluir dentro de la herramienta digital, permitiendo un aprendizaje autónomo y significativo basado en la tecnología, además de establecer una visión crítica de la realidad que envuelve al alumnado. De hecho, la búsqueda de información para la elaboración de los diferentes apartados de la revista digital, permite desarrollar un pensamiento científico crítico y que se adquiera destreza en el razonamiento deductivo e inductivo. Además, es considerada como una herramienta útil, innovadora y atractiva que puede ser usada tanto de manera monográfica para profundizar en el conocimiento de conceptos concretos trabajados en el currículum; así como de manera interdisciplinar y como proyecto colaborativo a lo largo de un período de tiempo prolongado integrando el trabajo desde diferentes especialidades. También es importante indicar que el uso de esta herramienta digital permite, según los propios alumnos, alcanzar múltiples y diversas competencias, especialmente la digital y la de aprender a aprender.

En conclusión, la elaboración de la revista digital como metodología activa de aprendizaje aplicada tanto para su lectura y, por tanto, de creación por parte del profesorado, como a la creación de la misma por parte del alumnado ha sido valorada muy positivamente prefiriéndola a las clásicas clases magistrales u otras herramientas digitales más conocidas.

\section{Referencias}

7. AGUILAR, M. (2012). “Aprendizaje y Tecnologías de la Información y Comunicación : Hacia nuevos escenarios

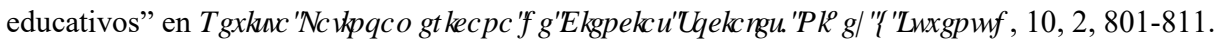

8. AMORES, Antonio Jesús (2019). "Las Nuevas Tecnologías como factor de motivación”. \&DPSXV( GXFDFlyQD UHLWDG JLWDORFHQUI Vol. 16, pp.39-44.

9. https://www.campuseducacion.com/revista-digital-docente/numeros/16/index.html\#43/z [Consulta: 21/03/2020] 
10. BUSQUET-DURAN, J., MEDINA-CAMBRON, A., BALLANO-MACIAS, S. (2013). "El uso de las TRIC y el choque cultural en la escuela. Encuentros y desencuentros entre maestros y alumnos" en 5 HLWDO HWUHU QHDCH \&RP XQFDFYQ 4, 2, 115-135.

11. HERNÁNDEZ, R.M. (2017). "Impacto de las TIC en la educación: retos y perspectivas" en 3 URSYUURVI 5HНHMHQWFIRQHИ 5, 1, 325-347.

12. NARANJO, M.L. (2009). "Motivación : perspectivas teóricas y algunas consideraciones de su importancia en el ámbito educativo" en 5HLWD( GXFDFyQ 33, 2, 153-170.

13. SOLBES, J., MONTSERRAT, R., FURIÓ, C. (2007). "El desinterés del alumnado hacia el aprendizaje de la



14. TELlO, J., AGUADED, J. I. (2009). "Desarrollo profesional docente ante los nuevos retos de las TIC en los centros educativos" en 3L[HD\%WW5 HLWDG GHO HARVI L( GXFDFyQ 34, 31-47. 